\title{
Angle division multiplexing in multimode fibers for optical board-to- board interconnection
}

\section{K.-H. Brenner, R. Klug, Ulrich Krackhardt}

K.-H. Brenner, R. Klug, Ulrich W. Krackhardt, "Angle division multiplexing in multimode fibers for optical board-to-board interconnection," Proc. SPIE 10296, 1999 Euro-American Workshop Optoelectronic Information Processing: A Critical Review, 102960E (2 June 1999); doi: 10.1117/12.365911

SPIE Event: Euro-American Workshop on Optoelectronic Information Processing, 1999, Colmar, France 


\title{
Angle division multiplexing in multi-mode fibers for optical board-to-board interconnection
}

\author{
K.-H. Brenner, R. Klug, U. W. Krackhardt \\ Lehrstuhl für Optoelektronik, Universität Mannheim, B6, 26, 68131 \\ Mannheim
}

\begin{abstract}
The propagation angle $\vartheta$ between the symmetry axis of a fiber and the principle propagation direction of a beam is conserved over short distances within a step-index multimode fiber. This conservation behavior can be used for multiplexed transmission by assigning different channels to different propagation angles. Furthermore, due to the reduction of the angular spread in the fiber, the temporal bandwidth is increased compared to multi-mode transmission. To realize this angular coding, suitable optical setups for multiplexing and demultiplexing operations were designed. The experimental results on the transmission capabilities of an angular multiplexed multimode fiber are presented.
\end{abstract}

Keywords: Optical Interconnection, Multimode fibers, Multiplexing

\section{INTRODUCTION}

Optical signal transmission benefits, among other features, from its huge potential of multiplexing. Besides wavelength, time or space division multiplexing (WDM, TDM, SDM) an alternative multiplexing technique is proposed here: Angular division multiplexing (ADM). This type of multiplexing uses the fact that the propagation angle $\vartheta$ between the symmetry axis of a fiber and the principle propagation direction of a beam is conserved over short distances within a step-index multimode fiber. This conservation behavior can be used for multiplexed transmission by assigning different channels to different propagation angles. Furthermore, due to the reduction of the angular spread in the fiber, the temporal bandwidth is increased compared to multi-mode transmission. Thus other multiplexing methods like TDM, WDM may be used in addition. The fundamental properties of ADM have already been developed 20 years ago ${ }^{1,2}$, where ADM was considered as a multiplexing technique for long distance communication. The results of these works showed that there is a trade-off between the number of channels and the propagation distance. Depending on the quality of the fiber, for distances larger than $100 \mathrm{~m}$, due to mode coupling, less than four channels could be realized. The mode coupling occurs because of inhomogeneities of the core index and because of possible roughness of the interface between core and cladding. The present situation is different from the situation 20 years ago in two aspects: Today the quality of multi-mode fibers has 
increased significantly. Furthermore there is a need for optical communcation in the shortdistance regime. With processors approaching the $1 \mathrm{GHz}$ clock frequency range, optical interconnections have become competitive also for medium and short distances. For distances in the board to board regime, multimode fibers are preferable, since fabrication costs are an important issue. Multi-mode fibers are largely insensitive to misalignment and to environmental changes and can be fabricated in polymers (POF) at very low cost. For multi-processor systems, interconnects with high bandwith and low latency are required. With ADM the bandwidth in multi-mode fibers is significantly higher than in single-channle operation. Thus the demands for processor interconection can be met.

To realize ADM, suitable optical set-ups for multiplexing and demultiplexing operations were designed. The experimental results on the transmission capabilities of an angular multiplexed multimode fiber are presented. In section 2. The fundamental properties of ADM are reviewed and estimates of the achievable number of channels are given. In section 3 optical set-ups are proposed for multiplexing and for de-multiplexing. A specially designed diffractive optical element serves as a mapping device between angular spectrum and spatial positions in the detector plane. The experimental results on the transmission capabilities of an angular multiplexed multimode fiber are presented in section 4.

\section{ANGULAR PROPERTIES AND BANDWIDTH OF ADM}

For short fiber transmission lines, i.e. over distances in the order of a meter, the coupling angle $\vartheta$ between the symmetry axis of a fiber and the principle propagation direction of a beam is conserved very well. Fig. 1 indicates that the cone angle at the exit of a multimode fiber is identical to the coupling angle at the input.

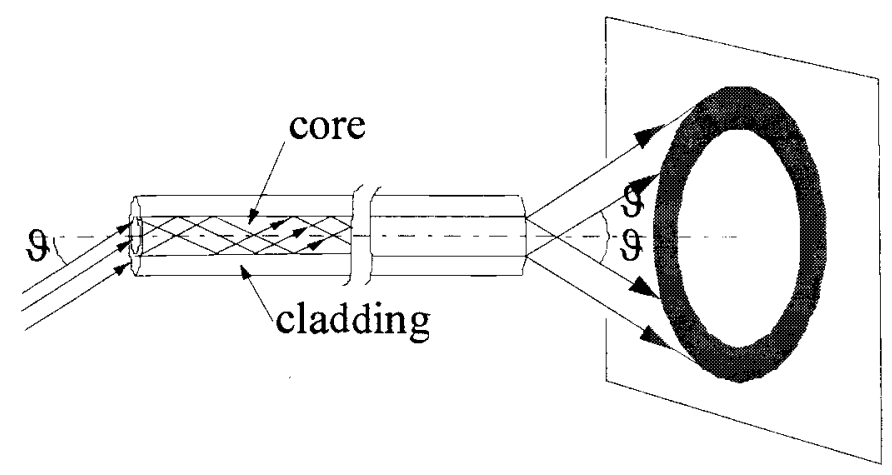

Fig. 1: Conservation of coupling angle $\vartheta$

Fig. 2 shows a measured output pattern of a step-index multi-mode fiber resulting from collimated beams coupled into the fiber under different directions separated by $\delta \vartheta=70$ mrad. Among the 12 channels that can be distinguished, only every second channel was illuminated. The intensity in the output plane of the fiber is distributed over an annular rings, the radius of a circle corresponds to the coupling angle $\vartheta$. 




Fig. 2: Output pattern with 6 from 12 channels enabled

The finite width of the circle results from two effects:

1) Due to the uncertainty principle the finite diameter $d$ of the fiber core results in a finite angular spectrum (finite $\vec{k}$-spectrum) of the coupled beam. The angular spread is determined by the wavelength $\lambda$ and the core diameter $d$.

2) 9-blur due to fiber interaction: Imperfections of both the fiber core and the core/cladding interface induce mode coupling, described ${ }^{2}$ by a mode coupling constant $D$. Since this effect scales with the propagation distance, the angular blur increases with the fiber length. Additionally, these imperfections can be introduced by mechanical stress of the fiber, e.g. by bending or squeezing.

Consequently the number of channels due to finite $\vec{k}$-spectrum of the coupled beam is given by the equation:

$$
N=\frac{\theta}{\Delta \theta} \quad \Delta \theta=\frac{\lambda}{4 d}
$$

The angular spread due to mode coupling can be described by a power cupling equation ${ }^{1}$

$$
\frac{\partial P(\theta, z)}{\partial z}=-A \theta^{2} P+\frac{D}{\theta} \frac{\partial}{\partial \theta}\left(\theta \frac{\partial P}{\partial \theta}\right)
$$

where the mode coupling constant $\mathrm{D}$ is given by

$$
D=d_{0}\left(\frac{\lambda}{4 n d}\right)^{2}
$$

The equation can be solved analytically:

$$
P(x, z)=\frac{e^{-\frac{x_{0}+x}{2}\left(\frac{1+\exp (-b z)}{1-\exp (-b z)}\right)} \cdot e^{-b z / 2}}{1-\exp (-b z)} I_{0}\left(\sqrt{x x_{0}} \frac{2 e^{-b z / 2}}{1-\exp (-b z)}\right)
$$


with

$$
x=\theta^{2} \sqrt{A / D} \quad b=4 \sqrt{A D}
$$

resulting in an expression for the power as function of angle and propagation distance, which is proportional to a Bessel-function $I_{0}$ of second kind. To illustrate the potential for multiplexing, the optical intensity as a function of coupling angle is shown in fig. 3 . Since the analytical result is only a function of the product of mode coupling constant $D$ and the propagation distance $z$, the figure is shown for different parameters $z \cdot D$, with the thick line highlighted as the case $1000 \mathrm{z} D=1$.

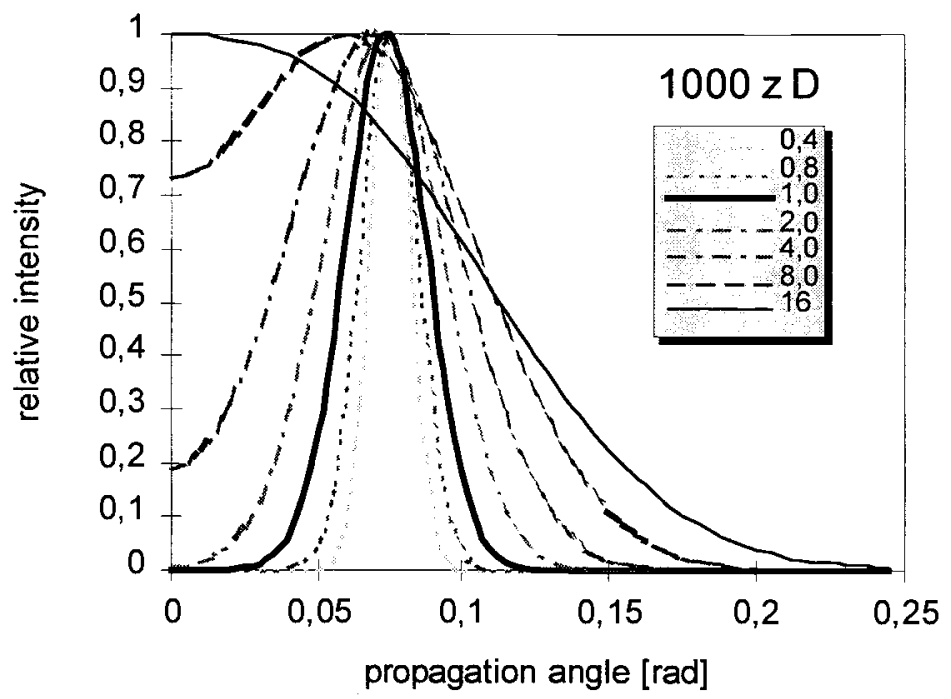

Fig. 3 Optical intensity as a function of coupling angle

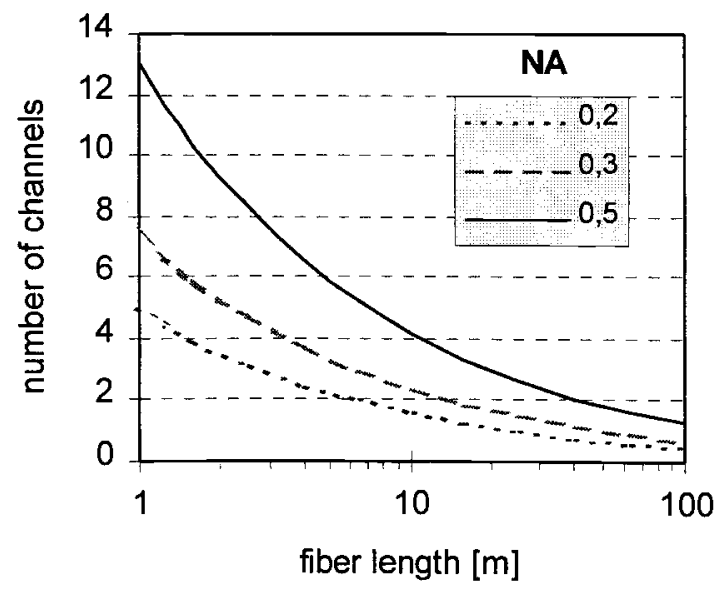

Fig. 4 Achievable number of channels as a function of propagation distance 
Fig 4. Illustrates how the achievable number of channels depends on propagation distance and the numerical aperture NA of the fiber. The calculation was performed for a typical value of the mode coupling constant of $D=3 \cdot 10^{5} \frac{\mathrm{rad}^{2}}{\mathrm{~m}}$. It indicates that 12 channels can be operated for an interconnect distance of $1 \mathrm{~m}$ and a multi-mode fiber with an NA of 0.5 . The bandwidth of multi-mode fibers is limited by mode dispersion according to

$$
\omega_{\max }=\frac{2 \pi c}{\left(n_{f}-n_{c}\right) \cdot L}
$$

For large fiber lengths $L$ and large NA-fibers with a high index difference $n_{f}-n_{\mathcal{C}}$ between fiber core and cladding, the maximum frequency is only in the $100 \mathrm{MHz}$-range. It is very interesting to note, that in the case of ADM the bandwidth grows with the number of multiplex channels $N$ according to

$$
\omega_{A D M}=N \omega_{\max }
$$

This unusual behavior can be explained as follows. Since one channel occupies only $1 / \mathrm{N}$ of the full angular range, the time delay between the minimum and maximum angle also decreases with $N$ and the bandwidth consequently increases with $\mathrm{N}$. For the fiber used in our experiments, the bandwidth of each channel can be $2 \mathrm{GBps}$, resulting in an overall digital bandwidth of $32 \mathrm{GBps}$ for 16 channels.

\section{OPTICAL MUX - AND DE-MUX - DEVICES}

The conservation properties described in section 2 can be used for realizing a multiplexed fiber transmission line in such a way that different logical channels are encoded by different coupling angles 9 . As pointed out in section 2 , the maximum number $N$ of multiplexed channels is basically limited by cross-talk due to modecoupling. Therefore, the parameters to optimize are the fiber length $\mathrm{L}$, the core diameter, the fiber NA and the mode-coupling constant $D$, which can only be influenced by selecting appropriate fiber types. The multiplex-and demultiplex devices then have to be adapted to the fiber parameters.

Fig. 5 shows a possible implementation of a MUX-device: Each output of the fiber is first collimated by a corresponding micro lens. The collimated beams are then focused onto a common spot by a macro lens or a section of a macro lens. The angular separation can be adjusted by the diameter of the micro lenses and the focal length ratio of micro and macro lens. 


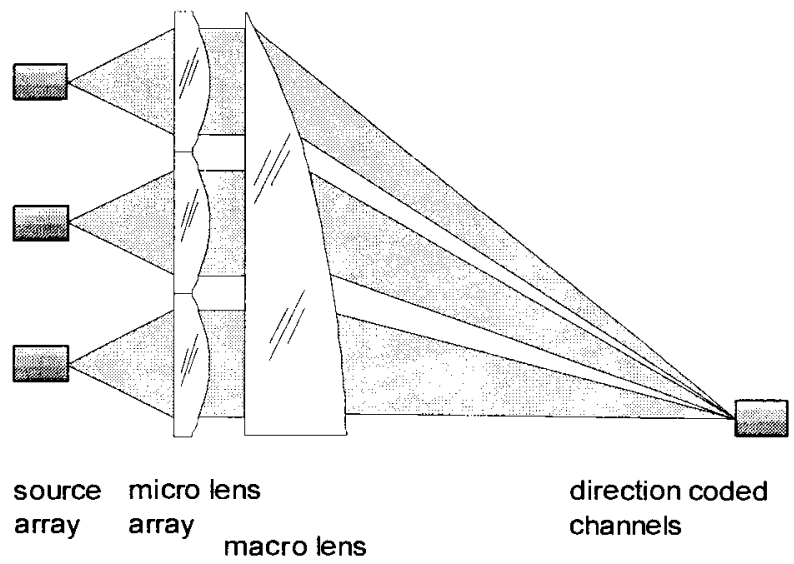

Fig. 5 Concept for a MUX-device, coupling different low-NA fiber channels into a high NA multimode fiber

The DeMUX device maps light distributed over different annular rings (corresponding to different $\vartheta$-directions) to separated points in the detector plane. The device consists of a lens, which images the fiber output to the detector plane and a filter to perform the mapping between annular rings and positions on the detector plane. Basically this filter is a set of prisms, each clipped by an annular ring. Shape and location of the rings are defined by the intensity distribution generated at the filter plane. Within each ring the deflection angle is constant and tuned to match the corresponding detector position. Fig. 6 shows an example where the detectors are lined-up. An extension of the concept to a planar array of detectors only requires a different filter design and is straightforward.

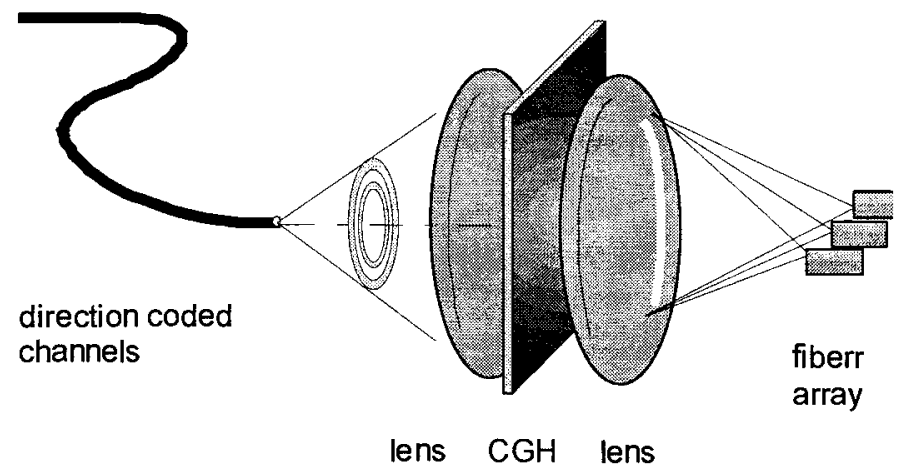

Fig 6: Concept for a DeMUX-device.

Different annular zones are assigned to regions on the $\mathrm{CGH}$, which directs the light to different output locations.Because of design flexibility we decided to use a computer generated diffractive element (CGH). To achieve high diffraction efficiency $\eta$ and low 
stray light (i.e. low cross-talk) an 8 -level $\mathrm{CGH}$ with $\eta \approx 80 \%$ is fabricated using lithography and reactive ion etching (RIE). Such a deflecting component can be designed for almost arbitrary detector geometries. It would even be possible to include fan-out operations.

\section{EXPERIMENTAL RESULTS}

To experimentally verify the conservation behavior of a fiber transmission line a stepindex multi-mode fiber is used with a core diameter of $d=200 \mu \mathrm{m}$, an NA $=0.48$ and a length of $\mathrm{L}=40 \mathrm{~cm}$. The thickness of the annular rings in Fig. 2 is measured by a clipping operation with a threshold of $(1 / \mathrm{e}) 2$ with respect to the maximum intensity. In terms of angles, the annular rings extend over a range of $\delta \vartheta=35 \mathrm{mrad}$. The fiber was excited with a He-Ne laser with a divergence of $1.2 \mathrm{mrad}$. Fig. 7 shows both conservation and spread of coupling angles $\vartheta$ at the output of the fiber. From fig. 7 one can design MUX and DeMUX devices with allowable cross-talk.

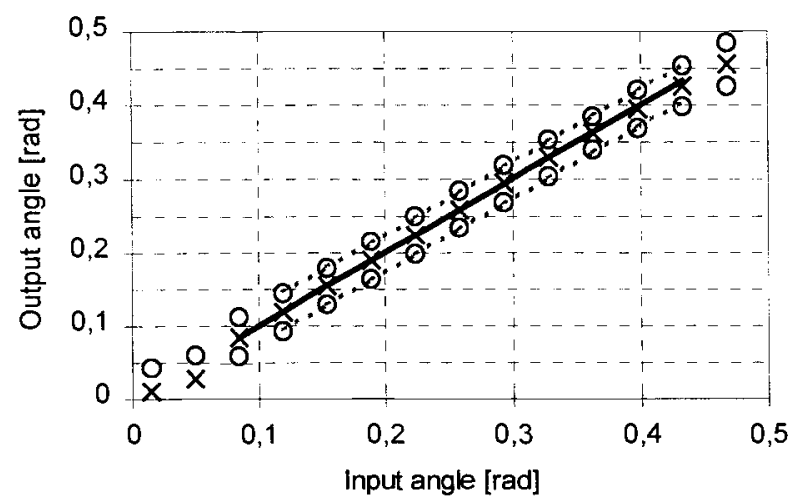

Fig. 7: Measurement of output angle vs. Input angle. The intensity center is marked by the solid line, the $1 / e^{2}$ positions by the dashed lines.

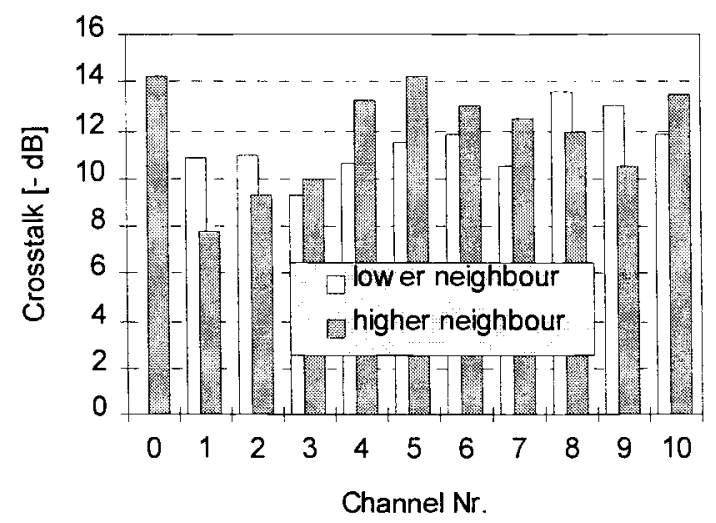

Fig. 8: Measured cross-talk for different channels 
In our example we considered a cross-talk of $-10 \mathrm{~dB}$ between adjacent channels to be tolerable, so we planned $\delta \vartheta=37 \mathrm{mrad}$ angular separation. The multiplexing capabilities of the fiber transmission line have been measured by using an optical DeMUX-unit that images the different channels onto a line. Fig. 8 shows the experimental result on measurements of cross-talk between adjacent channels. The average cross-talk value over all 11 measured channels is $-11 \mathrm{~dB}$. A higher number of multiplexed channels or lower cross-talk requires the use of fibers with higher NA and smaller coupling constant D.

\section{APPLICATION TO A FULLY INTERCONNECTED SYSTEM}

Fig. 9 illustrates a possible optical interface for each processing element PE in a bus configuration. The demultiplexer splits the optical channels in the fiber and directs them to a detector array. Each channel is assigned to a different processor. Thus each PE is able to receive input from all other PEs. At the output side, the input signals $n \neq M$ are regenerated and multiplexed into the outgoing multimode fiber whereas channel $M$ is used for the own output of the Mth PE. Since each PE has its own channel for output, no collision protocol is necessary for transmission. At the receiver side there also no conflict, since each interface has $\mathrm{N}$ detectors assigned to each PE. There may, of course, be bandwidth limitations if one PE receives simultaneously from several other PEs and is too slow to respond. This conflict can, however, be resolved either by buffering or by using other common protocols. The channel assignment is selected according to the output channel. Therefore, processor $M$ uses channel $M$ for its output. Since each processor can receive traffic from all other PEs $1 . . \mathrm{N}$ it is in particular possible for processor $M$ to receive its own output as well.

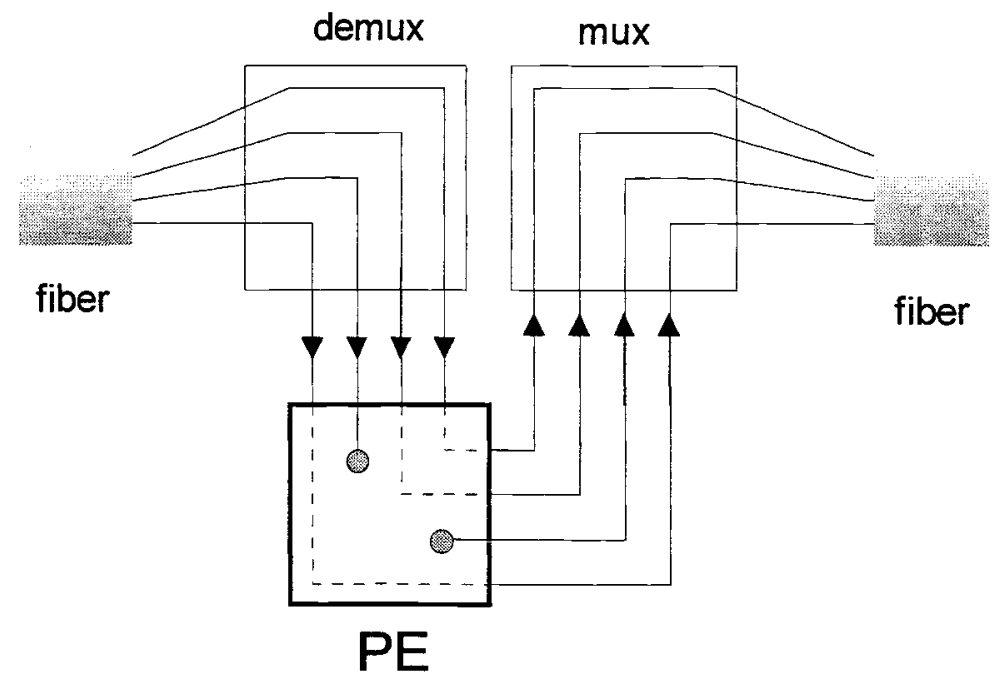

Fig. 9 Optical interface for each processing element $P E$ 
1999 Euro-American Workshop on Optoelectronic Information Processing / 69

\section{CONCLUSION}

Besides the well-known techniques for signal multiplexing in optical signal transmission there is an additional degree of freedom, the propagation angle, which can be used for multiplexing as well, since it is conserved in fibers over short distances. The advantage of this technique is that the information throughput in a fiber can be increased using only one wavelength and very little additional hardware. Furthermore, since each channel covers only a few optical modes, there is less modal dispersion as compared to transmission of one single channel through the same fiber without ADM. We have shown that input beams of different angles of incidence are imaged to circles of different radii in the output plane. This allows a separation of the individual multiplexed channels. The transmission behavior has been experimentally verified. A proposal for a particular demultiplexing device has been given and its feasibility was demonstrated. A number of 12 channels is possible now with existing fibers. By special tailoring of the numerical aperture and diameter of fibers a larger degree of multiplexing will be possible.

\section{REFERENCES}

1. U. Levy, H. Kobrinsky, A. Friesem: „Angular Multiplexing for Multichannel Communication in a Single Fiber", IEEE J. Q.El., QE-17, 2215-24 (1981)

2. G. Glode, „Optical Power Flow in Multimode Fibers“, Bell Syst. Techn. J. 51, 1767-83 (1972)

3. K.-H. Brenner, U. Krackhardt, R. Klug, "Directional multiplexing for optical board to board interconnections", Optics in Computing OC '98, Brugge, Belgium, SPIE

Vol. 3490 , p. 416 